\title{
Laparoscopic surgery in the treatment of incarcerated indirect inguinal hernia in children
}

\author{
YIYU YIN, HONGWEI ZHANG, XIANG ZHANG, FANG SUN, \\ HUAXIN ZOU, HUI CAO and CHENG WEN \\ Department of Pediatric Surgery, Xuzhou Children's Hospital, Xuzhou, Jiangsu 221002, P.R. China
}

Received April 4, 2016; Accepted September 27, 2016

DOI: $10.3892 /$ etm.2016.3830

\begin{abstract}
We aimed to explore the feasibility and the safety of the laparoscopic surgery for incarcerated indirect inguinal hernia (IIH) in children. From January 2012 to December 2014, 64 children were enrolled into this study. All 64 patients received laparoscopic surgery and we reviewed their perioperative and postoperative follow-up studies. In addition, we enrolled 60 cases of children who received traditional surgery of IIH administered through minimally invasive surgery as the control group. Results from the present study showed that the mean operation time for the laparoscopic group was 41.5 min (range, 15-80 min) which was significantly shorter than the control group. Nine cases developed incarcerated intestine necrosis, expanded umbilical incision and parallel resection anastomosis. They received laparoscopic hernia sac high ligation. Only 5 cases developed scrotum edema after the surgery. The postoperative length of the stay ranged from 2 to 7 days (average, 3.2). The postoperative follow-up was from 6 months to 1 year and no relapse or secondary testicular atrophy was observed in the laparoscopic group. The operation time, incidence of postoperative complications and length of stay in the laparoscopic group were decreased compared to the control group, and differences were statistically significant $(\mathrm{P}<0.05)$. In conclusion, laparoscopic surgery treatment for incarcerated inguinal hernia is safe and feasible and produced better results compared with the alternative.
\end{abstract}

\section{Introduction}

Indirect inguinal hernia (IIH) is the most common disease in pediatric surgery with an incidence ranging from 1 to $5 \%(1,2)$. In all cases of indirect hernia in children, the condition

Correspondence to: Dr Cheng Wen, Department of Pediatric Surgery, Xuzhou Children's Hospital, 18 Sudibei Road, Xuzhou, Jiangsu 221002, P.R. China

E-mail: wen_cheng1212@163.com

Key words: pediatric, inguinal hernia, indirect hernia, incarcerated hernia, laparoscopic manifests itself mainly by incarceration in approximately $1 / 6$ of all indirect inguinal cases (3).

Incarcerated IIH is very common in pediatric emergency surgery. In cases with shorter incarceration time, no obvious abdominal distension and no red or swollen scrotum and groin area, of manual reduction were used. In cases with longer incarceration time with red and swollen scrotum and groin area or conditions of low intestinal obstruction and presence of bloody stools in local scrotum, children required emergency surgery instead of manual reduction. Several studies on the application of laparoscopic surgery of incarcerated abdominal external hernia is available (4-6).

With the advancements of laparoscopy in Chinese pediatric surgery, we treated 64 cases of incarcerated IIH with laparoscopic surgery at the Xuzhou Children's Hospital (Jiangsu, China), and obtained better clinical effects compared with traditional methods.

\section{Materials and methods}

General information. From January, 2012 to December, 2014, 64 children were enrolled into the present study. These patients received laparoscopic surgery at the Department of Pediatric Surgery, and we reviewed their perioperative and postoperative follow-up studies. There were 43 males and 21 females, aged 3 months to 6 years (average, 2.1 years). The time of finding the groin mass ranged from 9 to $68 \mathrm{~h}$ (average, $20.8 \mathrm{~h}$ ). We had 33 cases of right side, 15 cases of left side and 16 cases of bilateral inguinal indirect hernia with only one side incarceration. Clinical manifestations included vomiting, paroxysmal crying in infants and complaining about lower abdominal pain in preschoolers. Before surgery, the children were examined using abdomen standing film to exclude gastrointestinal perforation and ultrasonography of groin area was performed to further confirm hernia contents.

Selection criteria used for the laparoscopic group were: i) no bloody stools; ii) no obvious abdominal distension; iii) generally in good condition to tolerate pneumoperitoneum; iv) little or no red and swollen groin area; and v) preoperative abdomen standing film with no gastrointestinal perforation, abdominal ultrasound without many ascites.

The control group comprised 60 cases of children treated with traditional surgery. Table I shows the comparison between the two groups. 
Table I. Comparison of the general information between the two groups of children.

\begin{tabular}{|c|c|c|c|c|c|c|c|c|}
\hline Groups & $\mathrm{n}$ & Age (years) & $\begin{array}{l}\text { Right } \\
\text { side (n) }\end{array}$ & $\begin{array}{c}\text { Left } \\
\text { side }(n)\end{array}$ & Bilateral (n) & Male (n) & Female (n) & $\begin{array}{c}\text { Cases of } \\
\text { intestinal } \\
\text { necrosis (n) }\end{array}$ \\
\hline Laparoscopic group & 64 & $2.10 \pm 0.18$ & 33 & 15 & 16 & 42 & 22 & 9 \\
\hline Control group & 60 & $1.90 \pm 0.16$ & 36 & 12 & 12 & 45 & 15 & 12 \\
\hline $\mathrm{t}\left(\chi^{2}\right)$ value & & $\mathrm{t}=0.805$ & \multicolumn{3}{|c|}{$\chi^{2}=0.907$} & \multicolumn{2}{|c|}{$\chi^{2}=1.300$} & $\chi^{2}=0.776$ \\
\hline P-value & & 0.423 & & 0.635 & & \multicolumn{2}{|c|}{0.254} & 0.378 \\
\hline
\end{tabular}

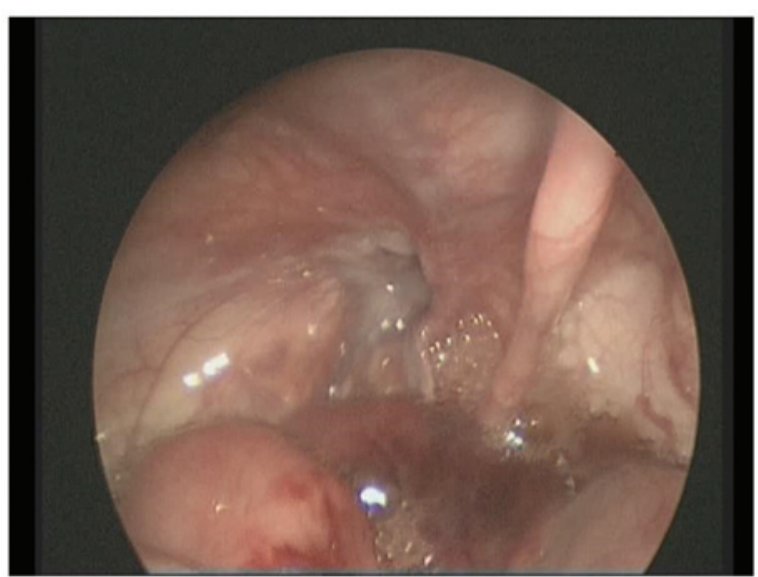

Figure 1. Returning intestinal canal.

Study approval was provided by the ethics committee of Xuzhou Children's Hospital. Written informed consent was provided by the children's family and/or guardians.

Methods. Patients were placed in a supine position, lower limbs were slightly outreached, and general anaesthesia was performed with tracheal intubation. Longitudinal incision was performed on the skin around the navel area to widen the navel ring. Peritoneal incision was performed and a 5-mm trocar was placed under direct vision to establish pneumoperitoneum (8-10-mm Hg pressure, placed $30^{\circ}$ laparoscope). Then a 3-mm deep incision on the left-mid abdomen was performed and $3 \mathrm{~mm}$ grasping forceps were placed. The operating table was adjusted to lower head higher feet posture and the patient's affected side was elevated. At that moment we could see clearly the internal ring of the affected side and the intestine or uterus in females, with attachment hernia. During surgery, patients received muscle relaxant and offered manual reduction under direct vision. Hernia contents were reconstructed in 44 cases successfully. In 13 cases we were not able to put back the hernia contents. In those cases, at the connection point of hernia blockage and abdominal wall, an incision was performed at approximately $0.5 \mathrm{~cm}$ to the upper edge of the hernia block. After mosquito vascular provoked anterior wall of the inguinal canal, we incised approximately $0.2-0.4 \mathrm{~cm}$. Consequently we applied manual reduction under direct vision again and reconstructed the intestine successfully (Fig. 1).

Internal ring closure method. Percutaneous tissue was sutured with the epidural needle on both sides, inside (Fig. 2) and

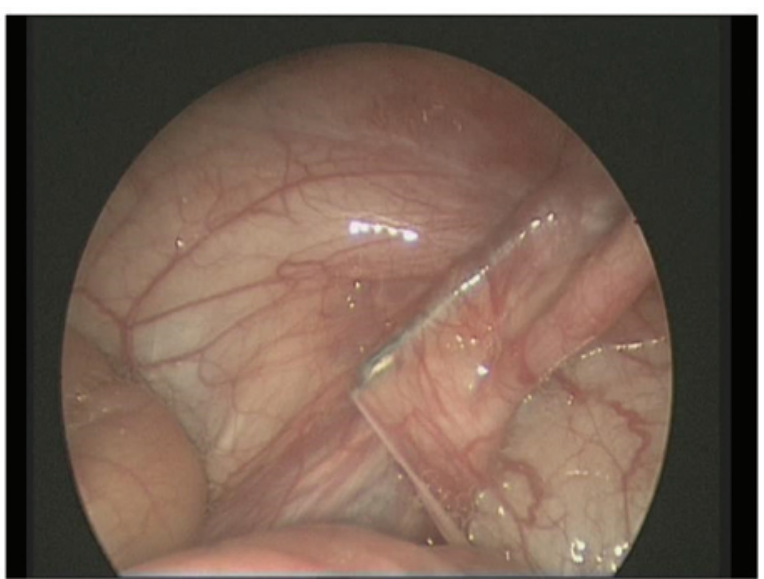

Figure 2. Suture on the inside of the inner ring.

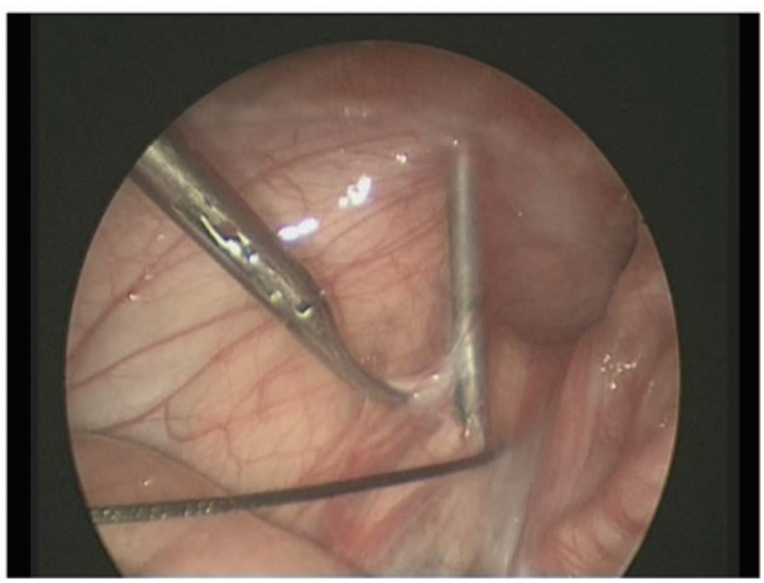

Figure 3. Suture on the outside of the inner ring.

outside (Fig. 3) at the mouth of the inner ring and a single line and two-wire brought through the pinhole. We used a two-wire loop to lead a single line out of the body and ligated inner ring after making a knot (Fig. 4). During the hernia ring suturing process, we incised the inguinal canal's anterior wall and passed two needles through the incised anterior wall of groin in order to tighten the outer opening after making a knot (Fig. 5).

Small intestine necrosis processing method. We expanded the navel incision and pulled the necrotic bowel out of the abdominal cavity. Then we treated it with resection and anastomosis 


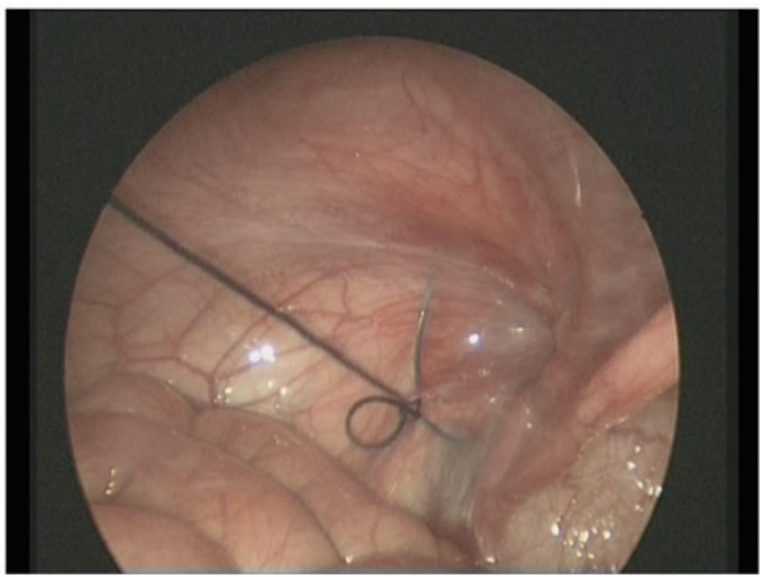

Figure 4. Taking out the single line in vitro with a loop.

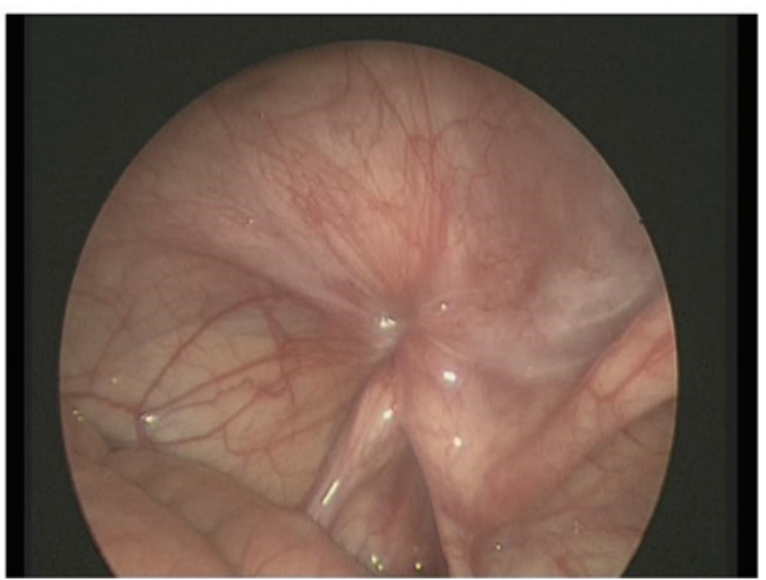

Figure 5. Inner ring after closing.

after the closure of mesangial hole. Intestine was then put back into abdominal cavity to re-establish pneumoperitoneum (hernial sac ligation step was the same as mentioned before). In the case of contralateral recessive hernia, family members were consulted again during the operation in order to obtain their consent for the surgery.

Postoperative treatment. Children without intestinal necrosis started drinking small amounts of water $6 \mathrm{~h}$ after the operation. In the absence of gastrointestinal reaction, children started liquid diet and were discharged 1-3 days after opera- tion. Children with chronic intestinal necrosis fasted after operation and were treated with gastrointestinal decompression for 3 days. They were fully rehydrated at the same time. They gradually started liquid or semliquid food after 3 days. They all received postoperative anti-infection treatment for 3 to 5 days. Antibiotics were stopped when no abnormal blood was observed and they were discharged 5-7 days after operation.

Statistical analysis. SPSS 13.0 statistical software (Chicago, IL, USA) was used for statistical analyses. Data are presented as mean \pm SD. For comparison we used the t-test or $\chi^{2}$ test. $\mathrm{P}<0.05$ showed statistically significant differences.

\section{Results}

The operation time in laparoscopic group ranged from 15 to $80 \mathrm{~min}$ (average, $41.5 \mathrm{~min}$ ). Five cases suffered from mild edema of scrotum after operation and 2 cases had inguinal subcutaneous congestion. Symptoms disappeared after raising scrotum and local physiotherapy. Postoperative length of stay ranged from 2 to 7 days (average, 3.2 days). All the patients were out-patients and were followed up for 6 months to 1 year (average, 8.7 months), there were no recurrence of indirect hernia in the group. No concurrent hydrocele or testicular atrophy in male children was observed.

The operation time of for control group ranged from 30 to $95 \mathrm{~min}$ (average, $53.9 \mathrm{~min}$ ). After the operation, we had 38 cases with obvious edema of scrotum and three cases with wound infection. Length of stay ranged from 4 to 7 days (average, 5.3 days) and they were followed-up to 6 months. We had 2 recurring cases (males), who agreed to the second surgery, 3 and 6 months after operation, respectively. For their second surgery we used the laparoscopic hernia sac high ligation method. Sixty cases of children were followed up from 6 months to 3 years (average, 1.5 years) and no secondary testicular atrophy or concurrent hydrocele in male children was observed (Table II).

\section{Discussion}

Pediatric incarcerated $\mathrm{IIH}$ is a common emergency treatment in pediatric surgery (mostly in infants). Many children have this condition for a long time before visiting a doctor. This can reduce the chance of a successful manual reduction and leads the medical team to opt for surgery. Traditional release of incarcerated hernia surgery needs to dissect the inguinal

Table II. Situation of perioperative period and complications in the two groups.

Postoperative complications

\begin{tabular}{|c|c|c|c|c|c|c|}
\hline Groups & $\mathrm{n}$ & $\begin{array}{c}\text { Time of } \\
\text { operation }(\mathrm{min})\end{array}$ & $\begin{array}{l}\text { Length of } \\
\text { stay (days) }\end{array}$ & $\begin{array}{c}\text { Edema of } \\
\text { scrotum (n) }\end{array}$ & Recurrence (n) & $\begin{array}{c}\text { Incision } \\
\text { infection (n) }\end{array}$ \\
\hline Laparoscopic group & 64 & $41.5 \pm 15.9$ & $3.3 \pm 1.79$ & 5 & 0 & 0 \\
\hline Control group & 60 & $53.9 \pm 13.8$ & $5.3 \pm 1.08$ & 38 & 2 & 3 \\
\hline $\mathrm{t}\left(\chi^{2}\right)$ value & & $t=-4.630$ & $\mathrm{t}=-7.892$ & & $\chi^{2}=17.086$ & \\
\hline P-value & & $<0.001$ & $<0.001$ & & $<0.001$ & \\
\hline
\end{tabular}


canal therefore the probability of postoperative complications, such as edema of scrotum, hematoma, indirect hernia recurrence is high. This method of surgery requires a 2-3-mm operation incision. Laparoscopic surgery for inguinal hernia has been carried out for many years. This method has several advantages compared with the traditional method. Among the advantages are the small operation incision, small intraoperative injury, fast procedure and efficient, quick recovery with minimal impact on the patients' reproductive system (7). There are several studies (8-10) on performing laparoscopic technology for pediatric incarcerated IIH. Since 2011, our hospital has carried out two holes approach laparoscopic surgery treatment for incarcerated IIH and obtained satisfactory results. In the laparoscopic group we obtained better results compared to the control group. In the observation group, the operation time and length of stay was shorter while the incidence of postoperative complications was much lower compared with the control group.

Laparoscopic surgery treatment for incarcerated IIH offered several advantages: i) during the operation under anesthesia we supplied muscle relaxants; ii) after the reduction was carried out, we were able to directly observe the revascularization of hernia contents; and iii) in the case of no intestine necrosis, we obtained recovery almost as in the case of ordinary laparoscopic inguinal hernia surgery. The recurrence rate of laparoscopic incarcerated hernia surgery is usually lower than that of traditional surgery (5), and we obtained the same type of results in the present study. In traditional surgery, under anesthesia, the return of hernia contents can be a serious problem for the surgeon, because it is difficult to explore the abdominal inner intestine through groin incision. Some Chinese doctors have reported good results using laparoscopic monitoring on children with failed manual reduction surgeries. They used grasping forceps to lead downward or adopting electric hook or scalpel to incise the inner mouth in the abdominal cavity (11). This method had the risk of avulsion of intestinal serosa or injury of edema intestinal near the inner mouth. We used the approach of opening part of the front wall of the inguinal canal through abdominal stripe incision, which could turn back the intestine quickly and reduce the damage for hernia contents because of reset force. For treating the inner mouth after turning hernia contents were returned, we used epidural needle with thread method. This was conducted easily and added no extra financial burden on the families. This approach may be appropriate for underdeveloped and poor regions. The results revealed that our method did not increase the risk of postoperative recurrence, and did not need any extra equipment.

In conclusion, compared with traditional surgery, laparoscopic surgery in pediatric incarcerated IIH has numerous advantages, such as fast reduction, small damage, short time of operation and length of stay, and certain clinical application value.

\section{References}

1. Wiener ES, Touloukian RJ, Rodgers BM, Grosfeld JL, Smith EI, Ziegler MM and Coran AG: Hernia survey of the Section on Surgery of the American Academy of Pediatrics. J Pediatr Surg 31: 1166-1169, 1996.

2. Kapur P, Caty MG and Glick PL: Paediatric hernias and hydroceles. Pediatr Clin North Am 45: 773-789, 1998.

3. Mackinnon AE: Hernia and hydrocele. In: Pediatric Surgery. Vol 32. Atwell JD (ed). Arnold, London, pp309-312, 1998.

4. Saggar VR and Sarangi R: Endoscopic totally extraperitoneal repair of incarcerated inguinal hernia. Hernia 9: 120-124, 2005.

5. Hoffman A, Leshem E, Zmora O, Nachtomi O, Shabtai M, Ayalon A and Rosin D: The combined laparoscopic approach for the treatment of incarcerated inguinal hernia. Surg Endosc 24: 1815-1818, 2010.

6. Ferzli G, Shapiro K, Chaudry G and Patel S: Laparoscopic extraperitoneal approach to acutely incarcerated inguinal hernia. Surg Endosc 18: 228-231, 2004.

7. Endo M, Watanabe T, Nakano M, Yoshida F and Ukiyama E: Laparoscopic completely extraperitoneal repair of inguinal hernia in children: a single-institute experience with 1,257 repairs compared with cut-down herniorrhaphy. Surg Endosc 23: 1706-1712, 2009.

8. Tatli D and Numanoglu KV: Transverse testicular ectopia associated with incarcerated inguinal hernia: a case report. Cases J 1: 200, 2008.

9. Kaya M, Hückstedt T and Schier F: Laparoscopic approach to incarcerated inguinal hernia in children. J Pediatr Surg 41: 567-569, 2006.

10. Nah SA, Giacomello L, Eaton S, de Coppi P, Curry JI, Drake DP, Kiely EM and Pierro A: Surgical repair of incarcerated inguinal hernia in children: laparoscopic or open? Eur J Pediatr Surg 21: 8-11, 2011.

11. Bin S, Hui W and Yang P: Laparoscopic high ligation of hernial sac for the treatment of 18 cases with inguinal incarcerated hernia in Chinese. J Minim Invasive Surg 13: 84-86, 2013. 